\title{
OPERATIONAL REQUIREMENTS
}

\author{
Gianluigi Arduini \\ SL Division
}

\begin{abstract}
On the basis of the experience gained in running LEP with an increasing number of superconducting RF units under global voltage control, the operational requests on the RF control software to be used in the control room for the LEP 2 phase are discussed. The maximum attainable beam energies, taking into account the consequences of a trip of one or several RF units on quantum lifetime, are also presented.
\end{abstract}

\section{1 '95 OPERATIONAL EXPERIENCE}

Three aspects charcterizing 1995 LEP operation are considered relevant for analyzing the operational experience as regards the RF control from the Prevessin Control Room (PCR):

- the increase in the number of RF units and in particular the larger and larger use of superconducting (SC) units. Being the RF voltage provided by a $\mathrm{SC}$ unit more than twice that available from a conventional copper unit, any instability and in particular any RF trip has an evident impact on the beam. In some cases, particularly at high energy, when the reserve of the total RF voltage is smaller, an RF trip may result in a partial or complete beam loss. During the 1995 LEP 1 phase $27 \%$ of beam losses were due to an RF trip while in the LEP 1.5 phase this percentage increased up to $90 \%$. The frequent occurrence of RF problems determined a massive use of programs for the diagnosis and the control of the RF units.

- the routine use of the Global Voltage Control (GVC) software in operation. This program was designed in order to provide a tool for the operator to keep a desired total voltage and in minimizing RF asymmetries during ramp and in physics.

- the Energy Scan for the precise determination of the mass and the width of the $Z^{0}$ line. This required a complete and reliable logging of the RF parameters.

Essentially four main functionalities of the RF control system are of interest for operations and therefore were made available in PCR: RF fault diagnostics, RF switch on, GVC and RF logging. Their performances in the 1995 run are discussed below.

\subsection{RF fault diagnostics}

Fast RF fault diagnostics programs providing concise reports on the status of the RF hardware are essential to rapidly recover normal operation conditions. The localization of the faulty unit and the detection of the fault are necessary to determine which actions the operator must take to restart the unit and, in case of major problems, to provide the RF expert with all the information required for an effective intervention. The alarm and RF surveillance screens are the first reference for the operator when abnormal beam parameters, that can be attributed to a change in the RF voltage distribution, are observed. These screens already provide a concise indication of the faulty unit, the origin of the $\mathrm{RF}$ trip (RF or power converter interlocks) and the status of the RF unit. During the 1995 run no alarm was generated in case of a SC unit trip, furthermore the refreshing rate of the RF surveillance screen data was relatively slow (approximately once every 1-2 minutes).

Once detected the fault, it was often necessary to determine the interlock status of the RF unit before proceeding to restart it. While a summary of the RF and $\mathrm{HV}$ interlock status of any number of $\mathrm{Cu}$ units was available on Apollo under the operational environment (SloppySoft), a X-terminal expert program had to be used to have a similar report for the SC units. Being an expert program the latter lacked of a user-friendly interface and it was difficult to consult because it provided an interlock report only for one unit at a time and too many details for operational use.

\subsection{RF switch on}

RF switch on was often hampered by communication problems linked to the GPIB bus. Switch on with beam was definitely more problematic for SC units, because of the major complexity of the tuning procedure, as compared to $\mathrm{Cu}$ units. This procedure used the beam current as an input parameter to determine the optimum tuning power. Tuning of the SC cavities was then achieved by thermal expansion of Ni bars (cooled by $\mathrm{He}$ gas flow) rigidly connected to the extremities of the cavities and heated with coils. The unit switch on procedure took about 10 minutes (approximately equal to the time required to ramp from $44 \mathrm{GeV}$ to $65 \mathrm{GeV}$ ) as compared to about 1 minute for $\mathrm{Cu}$ units. The switch on program was not protected against beam current reading errors, only a protection against overheating of the 
tuning bars existed. These errors sometimes resulted in heating the tuning bars above the optimum value and therefore in a delay of the switch on by a few tens of minutes (the time taken by the tuning bar to cool down).

A program to switch on any number of units was available in PCR under SloppySoft, nevertheless the switch on status was not updated automatically but only on request. This feature proved to be of particular interest for the SC units because of the long switch on time as compared to $\mathrm{Cu}$ units. For this reason a $\mathrm{X}$ terminal program, providing a continuous update of the switch on status for the SC units and generally used by the RF expert to control the RF units, was made available by the RF group as a temporary fix to the above problem. The considerations expressed in section 1.1 about expert programs equally apply to this case.

\subsection{Global Voltage Control}

The Global Voltage Control program was successfully used since the beginning of the 1995 run to control the distribution of the RF voltage among the RF units during the ramp.

The following problems were observed during operation:

- unpredictable results were often observed when trimming the synchrotron tune $\mathrm{Q}_{\mathrm{s}}$ : in some cases the total voltage did not change, in some others (when a mismatch was found between the required total voltage and the effective voltage) an unrelevant $\mathrm{Q}_{\text {s }}$ trim $\left(1 \times 10^{-6}\right)$ was used to re-estabilish the total $\mathrm{RF}$ voltage. The operator was often left with the choice of disabling GVC and setting the RF voltage of the individual units or to change the total $\mathrm{RF}$ voltage in the GVC control program, with the result that no record was left of the trim done.

- in a few cases the voltages of some RF units were found out of tolerance (even higher than the maximum RF voltage settings) as compared to the required settings;

- the absence of a "set vector" option during the LEP 1.5 phase, when the injection energy was raised from $20 \mathrm{GeV}$ to $22 \mathrm{GeV}$, required the generation of the RF settings for $20 \mathrm{GeV}$ (vector 0 ) and then the ramp to $22 \mathrm{GeV}$ (vector 17).

The above problems, but in particular the lack of a user-friendly interface, are probably at the origin of the limited use of GVC in physics. At least 4 applications should be used to control the system and to produce displays, such as those showing the RF level of each unit or the RF voltage in the sectors left and right of each interaction point.

The operator could not specify the symmetry type he wanted to run the machine because this condition was hard-coded in the program. The symmetry type is an important parameter in the optimization of the performance of the machine, not only during the ramp, but in particular during physics.

During the LEP 1.5 phase frequent changes in the RF configuration were required in the quest for stable operation and in order to cope with the evolution of the performances of the individual units. In this respect editing and reloading the Current Data Set (the file containing, among other parameters, the maximum RF levels) required the use of two different applications and a lengthy procedure because of the absence of a graphic user interface (e.g. an input window).

\section{$1.4 R F$ data logging}

RF data logging during the Energy Scan was often affected by the communication problems already mentioned. As a result of that the logging of the RF voltages was discontinuous and required the constant intervention of the operator to reset communications. The absence, in the frame of the RF control software, of a dedicated application to check and reset communications with the RF units, further increased the number of applications required to run the RF hardware.

\section{RF SCENARIO}

During 1996 the number of SC units will further increase in two steps. The number of units and the maximum available total voltage foreseen in these two phases are listed in Tables 1 and 2, respectively. The equivalence $1 \mathrm{SC}$ unit $=2 \mathrm{SC}$ modules $=8 \mathrm{SC}$ cavities should be kept in mind in the following.

\begin{tabular}{|l|c|c|}
\hline & Number of units & $\begin{array}{c}\text { Max.RF voltage } \\
{[\mathrm{MV}]}\end{array}$ \\
\hline Cu cavities & 120 & 292 \\
\hline SC Cu/Nb cavities & 128 & 1280 \\
\hline SC Nb cavities & $4(12)$ & $34(102)$ \\
\hline \multicolumn{2}{|l|}{ Maximum total RF voltage [MV] } & $1606(1674)$ \\
\hline
\end{tabular}

Table 1: Installed RF cavities and maximum available RF voltage by week 22 (end of May). According to the $\mathrm{RF}$ installation plans the installation of 2 solid $\mathrm{Nb}$ modules (273.2 and 273.3) is not assured [1].

\begin{tabular}{|l|c|c|}
\hline & Number of units & $\begin{array}{c}\text { Max.RF voltage } \\
{[\mathrm{MV}]}\end{array}$ \\
\hline $\mathrm{Cu}$ cavities & 120 & 292 \\
\hline $\mathrm{SC} \mathrm{Cu} / \mathrm{Nb}$ cavities & 160 & 1600 \\
\hline SC Nb cavities & 16 & 136 \\
\hline \multicolumn{2}{|l|}{ Maximum total RF voltage [MV] } & 2028 \\
\hline
\end{tabular}

Table 2: Installed RF cavities and maximum available RF voltage by week 40 (end of September) [2].

The maximum RF voltages listed in Table 3 (corresponding to those used in operation in 1995) have been assumed for the individual units. These are slightly conservative values for the $\mathrm{Cu}$ units, in fact conditioning 
of these cavities is foreseen during the shutdown and an improvement of their maximum performances (by a few $\mathrm{MV} /$ unit) is expected [2].

\begin{tabular}{|l|c|}
\hline Unit type & Max. RF voltage [MV] \\
\hline $\mathrm{Cu}$ units (even numbers) & 38 \\
\hline $\mathrm{Cu}$ units (odd numbers) & 35 \\
\hline $\mathrm{SC} \mathrm{Cu}$ /Nb units & 80 \\
\hline $\mathrm{SC} \mathrm{Nb}$ units & 68 \\
\hline
\end{tabular}

Table 3: Maximum RF voltage per unit assumed in the calculation of the total available RF voltage in Tables 1 and 2 .

An estimate of the maximum beam energies achievable in 1996 with the expected total RF voltages listed in Tables 1 and 2 is presented in Table 4 for the $108^{\circ} / 60^{\circ}$ optics $\left(\alpha_{p}=1.38251 \times 10^{-4}\right)$. The reduction in the maximum achievable energy resulting by the consideration of a reserve in the RF voltage (in order to face the RF trip of one or two units) is also shown. The quantum lifetime drop in occasion of a SC unit trip is in fact so important (several order of magnitude) and the switch on procedure and GVC response time (about $1 \mathrm{~s}$ ) too slow that running without reserve would inevitably bring to loose the beam in case of a RF trip.

\begin{tabular}{|c|c|c|c|}
\hline & \multicolumn{3}{|c|}{ Maximum beam energy [GeV] } \\
\hline & no reserve & 1 klystron trip & 2 klystron trip \\
\hline June & $83.8(84.7)$ & $82.7(83.6)$ & $81.6(82.5)$ \\
\hline October & 88.9 & 88.0 & 87.1 \\
\hline
\end{tabular}

Table 4: Estimated maximum beam energy as a function of the reserve allocated for RF trips. In brackets are presented the values expected in case also the SC Nb modules 273.2 and 273.3 are installed by week 22 .

The maximum beam energy that allows a quantum lifetime larger than 100 hours has been assumed as a criterion in compiling Table 4 . The beam lifetime in collision is mainly dominated by beam-beam Bremsstrahlung (about 10 hours at LEP 2 [3]) therefore the above criterion is relatively conservative. Furthermore, Sands formula [4], that has been used in the above calculations, provides more pessimistic estimates of the quantum lifetime as compared to other formulas [5].

The maximum energies achievable with the $90^{\circ} / 60^{\circ}$ optics $\left(\alpha_{p}=1.90985 \times 10^{-4}\right)$ are about $0.4-0.5 \mathrm{GeV}$ lower than the corresponding ones listed in Table 4 for the $108^{\circ} / 60^{\circ}$ optics.

The voltage induced by the beam in a tuned SC cavity at high currents is not negligible (about -60 $\mathrm{MV} /$ tripped unit for a beam DC current of $8 \mathrm{~mA}$ [6]), nevertheless the cavities are rapidly detuned in the occurrence of a RF trip. Few tens of milliseconds are sufficient to detune the cavity by magnetostrictive action on the tuning bars and to reduce by one order of magnitude the impedance of the cavity seen by the beam and therefore the induced voltage. The further reduction in the quantum lifetime resulting from the negative induced voltage is therefore limited to the detuning time and it should not result in relevant beam losses.

\section{OPERATIONAL REQUIREMENTS}

As can be seen from Tables 1 and 2 the number of SC units will double by the end of May 1996 and will triplicate by the end of September 1996. A reliable and user-friendly RF control software is therefore mandatory. In formulating the operational requirements it seems reasonable to classify them in two sets, according to what is considered their degree of urgency.

\subsection{Improvement of the existing $R F$ control hardware and software}

The highest priority should be given to the solution of the communication problems because this would already have a beneficial effect on the speed and the effectivness of the switch on procedure, as well as on the reliability of the RF data logging.

The reliability and the versatility of the existing RF control software in the operational environment (SloppySoft) should be improved with the aim of minimizing the number of existing applications.

The possibility of checking and resetting communications should be made available under SloppySoft as before.

Alarms for every unit should be displayed in the PCR alarm screen and a faster update of the RF surveillance screen should be attained (update every $20 \mathrm{~s}$ should be feasible [7]). A more extensive use of colours should help in reducing the number of listed items (e.g., the Main Circuit Breaker status and the high voltage level could be presented in a single column, the same could be for the voltage loop status and the RF level) and should help the operator in the detection of the fault.

A HV and RF interlock summary should be available in SloppySoft for all the RF units.

The reliability and the speed of the switch on procedure should be improved, in particular protection against heating of the tuning bars above the optimum temperature should be implemented.

GVC reliability with respect to the points mentioned in section 1.3 should be enhanced. All the options foreseen for this applications should be implemented: the operator should have the possibility to choose among the different symmetry conditions and the auto-switch-on procedure with beam should be implemented [8]. Tripped units could be therefore automatically restarted after an automatic check of the fault type and switching on RF units during the ramp should be also feasible.

RF problems during the ramp could occur because of $\mathrm{RF}$ trips or vacuum problems on power couplers [9]. In 
that case stopping the ramp and restarting the tripped units could be a safe measure, taking into account that the switch on time for a SC unit is comparable with the time required to ramp from $22 \mathrm{GeV}$ to $80 \mathrm{GeV}$ without stopping. Therefore a 'stop of ramp' option should be provided in GVC (it could be even automatic in case of RF trip).

A 'set vector' option should allow more flexibility in changing the injection energy [10].

The possibility of taking into account the RF voltages of units that are on but not ramped proved to be useful already during the 1995 run and it would be important in choosing the ramp strategy and in the policy of keeping one or two units as a reserve of RF voltage in physics.

\subsection{Dedicated application for the RF control in $P C R$}

The degree of complexity of the RF hardware certainly justify the implementation of a dedicated application for the RF control under SloppySoft, such as those already existing for the separator and collimator control. This application should include:

- fault diagnostics, switch on program and communication manager for all the RF units, longitudinal and transverse feedback;

- user-friendly GVC manager permitting to select the above mentioned options (symmetry type, ramp strategy, vector number, ramp stop....) and to easily edit and reload the Current Data Set;

- displays: schematic synopsis with use of colours to indicate the status of a component and to interrogate easily the system (clicking on the displayed component), bar histograms displaying the RF voltage of each unit and the RF voltage in the sectors (these last two applications already exist);

- other diagnostics tools such as bunch display and bucket scan.

\section{CONCLUSIONS}

1995 operational experience with the RF control system has been characterized by the successful use of the Global Voltage Control during the ramp. Major concerns came from the unreliability of the communications with the hardware, the insufficient fault diagnostics for the SC units and the lack of flexibility of GVC to allow its efficient use in physics. The increase in the number of SC units in 1996 should allow the attainment of the maximum energies of $81.6 \mathrm{GeV}$ by the beginning of June and of $87.1 \mathrm{GeV}$ by the beginning of October, with an adequate reserve of RF voltage, for the $108^{\circ} / 60^{\circ}$ optics. In reason of the increased complexity and extension of the RF hardware, the improvement of the weak points above mentioned is mandatory for the start-up. The creation of a dedicated application for the RF control with its own fixed displays seems also justified once solved the more urgent problems above underlined.

\section{ACKNOWLEDGMENTS}

I would like to thank all the collegues of the LEP operation team for providing me with the results of their experience in operating the RF hardware from PCR and for their suggestions about the possible improvements. I am indebted to L. Arnaudon, D. Boussard, E. Ciapala, G. Geschonke and J. Sladen for the explanations concerning the RF hardware and controls, as well as the installation plans for 1996.

\section{REFERENCES}

[1] J. Montes, H. Schuhback, 'LEP - Planning de travaux pendant le SHUTDOWN du 27 NOVEMBRE 1995 au 16 JUIN 1996', 10 November 1995, Annexe 15.

[2] G. Geschonke, private communications.

[3] H. Burkhardt, 'Beam-Beam Tuneshift, Emittance and Lifetime', Proceedings of the Fourth Workshop on LEP Performance, J. Poole ed., CERN SL/94-06 (DI), Geneva, 1994, pp. 413-419.

[4] M. Sands, SLAC-121 (1970).

[5] F. Ruggiero, CERN SL/93-05 (AP).

[6] D. Boussard, '70 GeV Run - RF Considerations', transparencies presented at the 'Discussion Day on LEP Operation at $70 \mathrm{GeV}$ ', Ferney Voltaire, $11^{\text {th }}$ October 1995.

[7] E. Ciapala, private communication.

[8] E. Ciapala, 'How Can We Survive the 1995 RF Asymmetries ?', Proceedings of the Fifth Workshop on LEP Performance, J. Poole ed., CERN SL/95-08 (DI), Geneva, 1995, pp. 140-145.

[9] G. Geschonke, 'RF Constraints', Proceedings of the Fifth Workshop on LEP Performance, J. Poole ed., CERN SL/95-08 (DI), Geneva, 1995, pp. 195-197.

[10]G. de Rijk, 'How high we can push the injection energy of LEP ?', these Proceedings. 